Prof. Dr. rer. nat. Jürgen Vormann, Ismaning/München

\section{Nahrungsergänzungsmittel: Sinnvoller Beitrag zur Gesundheitsvorsorge}

Die Supplementierung unserer Ernährung mit verschiedensten Mikronährstoffen hat in den letzten Jahren zunehmend Bedeutung erlangt. Die Begründung hierfür ist insbesondere im Ausgleich einer unzureichenden Zufuhr mit der üblichen Ernährung zu suchen. Die Ursachen für die unzureichende Versorgung mit bestimmten Nährstoffen sind vielfältig, z.B.:

- unausgewogene Lebensmittelauswahl,

- Verarmung einzelner Lebensmittel an verschiedenen Mikronährstoffen,

- Verlust von Mikronährstoffen durch lange Transportwege und inadäquate Zubereitungsweise,

- erhöhter Bedarf bei verschiedenen pathophysiologischen Zuständen.

Um diese Nährstoffdefizite auszugleichen, finden Nahrungsergänzungsmittel Verwendung. Auch wenn bestimmte Nährstoffe direkt zur Behandlung von Krankheiten eingesetzt werden können und damit die Grenze zu Arzneimitteln überschritten wird, ist mit der Einnahme von Nahrungsergänzungsmitteln üblicherweise kein direktes therapeutisches Ziel verbunden; vielmehr sollen Stoffwechselfunktionen optimiert werden, wodurch vorzeitigem Auftreten von Krankheiten vorgebeugt wird.

Oft werden für die Beurteilung von Nahrungsergänzungsmitteln die gleichen Kriterien angewendet wie für Arzneimittel. Die gesundheitliche Bewertung von Lebensmitteln und Lebensmittelinhaltsstoffen unterscheidet sich jedoch erheblich von der Bewertung der Wirksamkeit von Arzneimitteln:

- Während Arzneimittel üblicherweise nur über einen zeitlich überschaubaren Zeitraum eingenommen werden und vor dem Beginn der Einnahme im Körper nicht vorhanden waren, wird mit Nahrungsergänzungen nur die verfügbare Menge schon vorher vorhandener Substanzen erhöht.

- Grundsätzlich werden Arzneimittel zur Behandlung von Kranken eingesetzt, während eine Nahrungsergänzung die Gesundheit langfristig erhalten und vorzeitigen Abnutzungserscheinungen vorbeugen soll.

Goldstandard für die Untersuchung der Wirksamkeit von Arzneimitteln ist die randomisierte, plazebokontrollierte Doppelblindstudie. Diese Untersuchungen werden naturgemäss an Kranken durchgeführt, bei denen der Effekt des Arzneimittels im Vergleich zu einem Scheinmedikament untersucht wird. Der Beobachtungszeitraum in diesen Untersuchungen ist meistens relativ überschaubar (Tage und Monate). Im Gegensatz dazu müssen die Effekte von Nahrungsergänzungsmitteln nicht bei Kranken, sondern bei Gesunden bewertet werden. Da nicht ein therapeutischer Nutzen beurteilt wird, sondern vielmehr diese Mikronährstoffe den allgemeinen Gesundheitszustand erhalten und vorzeitige Erkrankungen verhindern sollen, müssten diese Studien über sehr lange Zeiträume (Jahre und Jahrzehnte) durchgeführt werden. Hinweise auf die Bedeutung eines Lebensmittelinhaltsstoffs ergeben sich deshalb vor allem aus epidemiologischen und Fall-/Kontrollstudien. Allerdings kann aus diesen Untersuchungen keine strikte Kausalität abgeleitet werden, da neben den untersuchten Faktoren noch andere Inhaltsstoffe oder Lebensumstände für die gefundenen Effekte verantwortlich sein könnten.

Eines der Kriterien zur Bewertung des Sinns einer Nahrungsergänzung ist die Deckung des «Bedarfs» an einem Nährstoff. Aus der Geschichte der Ernährungswissenschaft ist bekannt, dass der «Bedarf» als die Zufuhr eines bestimmten Nährstoffs definiert wurde, die ausreicht, eine spezifische Mangelkrankheit zu vermeiden. Diejenige Menge, die die Mangelsymptome gerade noch verhindert, wurde als «minimaler täglicher Bedarf» definiert. Mit unterschiedlichen Sicherheitszuschlägen errechnet sich dann daraus eine bestimmte Zufuhrempfehlung. Das einfache Modell «ein Nährstoff - ein Mangelsymptom» entspricht jedoch nicht mehr dem gegenwärtigen Kenntnisstand. Man weiss heute, dass viele verschiedene Nähr-

\section{KARGER}

Fax +497614520714
(๑) 2010 S. Karger GmbH, Freiburg

Accessible online at: www.karger.com/szg
Prof. Dr. rer. nat. Jürgen Vormann

IPEV - Institut für Prävention und Ernährung

Adalperostrasse 37, 85737 Ismaning, Deutschland

Tel. +49 89-55267989, Fax -55267990

vormann@ipev.de 
stoffe gleichzeitig benötigt werden, um einen optimalen Stoffwechsel zu gewährleisten bzw. das Risiko von Krankheiten zu vermindern. Eine geringe Mikronährstoffzufuhr wird zuerst zu einer Erschöpfung vorhandener Speicher führen. Anschliessend kommt es zu Beeinträchtigungen biochemischer Abläufe, die am Anfang nur in subklinischen Symptomen erkennbar sind. Im weiteren Verlauf entwickeln sich unspezifische Symptome (z.B. verminderte Immunität), und erst nach längerer Zeit werden typische Mangelkrankheiten offensichtlich.

Bilanzuntersuchungen, wie sie unter anderem für Mineralstoffe durchgeführt wurden und als Basis für Zufuhrempfehlungen Verwendung fanden, sind oft nicht geeignet, den langfristigen Effekt einer höheren Mikronährstoffzufuhr zu bewerten. Wird von einem Mikronährstoff mehr ausgeschieden als zugeführt, so führt dieses sicherlich langfristig zu Mangelsymptomen. Eine ausgeglichene Bilanz besagt aber nur, dass dieser Mangel verhindert wird. Ob eine höhere Zufuhr dann einen darüber hinausgehenden Effekt hat, ist mit Bilanzuntersuchungen nicht festzustellen. Die zusätzlich zugeführten Mikronährstoffe sorgen transient für eine höhere Konzentration des jeweiligen Nährstoffs in bestimmten Körperkompartimenten (Darm, Blut, Ausscheidungsorgane). Mit dieser höheren lokalen Konzentration kann dann auch eine positive gesundheitliche Wirkung verbunden sein. Die oft geäusserte Behauptung, die Einnahme von Nahrungsergänzungsmitteln würde nur «teuren Urin» produzieren, ist deshalb nicht gerechtfertigt.

Eine andere Definition des «Bedarfs» entspricht besser dem gegenwärtigen Wissensstand als die reine Beschränkung auf die Vermeidung von Mangelsymptomen bzw. das Erzielen einer ausgeglichenen Bilanz: nämlich die Zufuhr, die über den weitaus überwiegenden Teil der Entwicklungsgeschichte des Menschen gegeben war.

In seiner jetzigen Form gibt es den Menschen seit circa 300000 Generationen. Den weitaus grössten Teil der Entwicklungsgeschichte haben unsere Vorfahren als Jäger und Sammler gelebt, denn erst vor circa 500 Generationen begann die Entwicklung der Landwirtschaft. Genetisch unterscheidet sich der Mensch der Gegenwart jedoch nur in sehr geringem Mass von den Menschen der Urzeit, d.h. die physiologischen und biochemischen Abläufe in unserem Stoffwechsel sind optimal an Lebens- und Ernährungsbedingungen angepasst, die in der Steinzeit vorherrschten. Die Ernährung des Steinzeitmenschen unterschied sich allerdings deutlich von der gegenwärtig üblichen. Unsere Vorfahren ernährten sich vornehmlich von Wildtieren und von gesammelten Früchten, Blättern, Nüssen und Samen. Nach der Erfindung und Entwicklung der systematischen Landwirtschaft veränderte sich das Lebensmittelangebot erheblich. Kalorien werden gegenwärtig überwiegend über Speicherkohlenhydrate (Stärke), industriell verarbeitete Pflanzenfette sowie relativ fetthaltige Nutztier- und Milchprodukte zugeführt. Der Anteil der frischen pflanzlichen Lebensmittel ging entsprechend erheblich zurück. Dies erklärt, dass die tägliche Aufnahme von Vitaminen, Mineralstoffen, Spurenelementen und sekundären Pflanzeninhaltsstoffen in der voragrarischen Zeit erheblich höher war, als es gegenwärtig der Fall ist. Da unsere physiologischen Abläufe sich aber schon in weit früherer Zeit entwickelten, sind sie an diese hohe Zufuhr von Mikronährstoffen adaptiert. Eine Nährstoffzufuhr wie in der Steinzeit ist deshalb für eine optimale Funktion unserer physiologischen Vorgänge biologisch besser angepasst.

Der Verbraucher kann heute durch umfangreiche Aufklärung über die Medien abschätzen, ob er sich gegenwärtig adäquat ernährt oder nicht. Allerdings haben auch intensive Aufklärungskampagnen nicht dazu geführt, z.B. die Zufuhr von Gemüse und Obst wesentlich zu erhöhen. Aber auch bei bewusster zeitweilig eingeschränkter Nährstoffzufuhr (z.B. bei Reduktionsdiäten) sollte der Verbraucher die Möglichkeit besitzen, den Mikronährstoffbedarf zu ergänzen.

Die Bestimmung eines Mikronährstoffdefizits ist mit den gegenwärtig verfügbaren Methoden (überwiegend Bestimmung einzelner Substanzen/Marker im Blut) leider sehr aufwändig und auch nicht immer aussagekräftig, da intrazelluläre Mangelsituationen auftreten können, ohne dass es zu einer Änderung der Blutkonzentration kommt. Der Aufwand einer detaillierten Analyse des individuellen Mikronährstoffstatus, um daraus eine Nahrungsergänzungsempfehlung abzuleiten, ist daher beträchtlich.

Wenn die Ernährungsanamnese eine unzureichende Zufuhr vermuten lässt, dann kann eine Nahrungsergänzung jedoch durchaus auch ohne vorherige Analytik erwogen werden. Selbstverständlich muss auch für Nahrungsergänzungsmittel abgewogen werden, ob die Einnahme mit Nebenwirkungen verbunden sein könnte. Solange die Dosis der Inhaltsstoffe diejenige Zufuhr nicht langfristig übersteigt, die auch durch eine optimale Lebensmittelauswahl erreicht würde, kann das Risiko jedoch vernachlässigt werden.

Prof. Dr. rer. nat. Jürgen Vormann, Ismaning/München 